\title{
Preface: Special Issue on Modeling, Simulation, and Optimization in Operational Research
}

\author{
Gerhard-Wilhelm Weber1,2 J. Joshua Thomas $^{3}$. \\ José Antonio Marmolejo Saucedo ${ }^{4}$ Ugo Fiore $^{5}$ • Igor Litvinchev ${ }^{6}$. \\ Pandian Vasant ${ }^{7}$
}

Published online: 8 September 2021

(c) Operations Research Society of China, Periodicals Agency of Shanghai University, Science Press, and Springer-Verlag GmbH Germany, part of Springer Nature 2021

"Operational Research" (OR) is "the discipline of applying advanced analytical methods to help make better decisions". By using techniques such as problem structuring methods and mathematical modeling to analyze nontrivial situations, OR gives executives the power to make more effective decisions and build more productive systems.

The host journal of this special issue, Journal of the Operations Research Society of China (JORSC), is the flagship journal of the Operations Research Society of China. Its primary goal is to promote researches and applications of all aspects of OR. JORSC provides a forum for practioners, academics and researchers in OR and related fields. It reflects the rapid social and economic development of China and leads to new problems and challenges which require new OR methodology and techniques. For closer information about JORSC, we refer to https://www.springer.com/journal/ 40305/aims-and-scope.

The objective of this special issue of JORSC has been to academically and scholarly gather the global research leaders, experts and scientists in the scientific and applied areas of Mathematical Modeling and Statistical Learning, Optimization and Data Science, Heuristics and Artificial Intelligence (AI) from all over the world to

\footnotetext{
Gerhard-Wilhelm Weber gerhard.weber@put.poznan.pl

J. Joshua Thomas jjoshua@kdupg.edu.my

José Antonio Marmolejo Saucedo jmarmolejo@up.edu.mx

Ugo Fiore

ufiore@unina.it

Igor Litvinchev igorlitvinchev@gmail.com

Pandian Vasant pvasant@gmail.com
} 
share their knowledge, experiences and insights on contemporary research achievements and future outlooks in these fields. This special issue has provided a "golden opportunity" for the international research community to interact and exchange their novel scientific results, innovative discoveries and important inventions among their colleagues and friends, many more readers and upcoming research partners. For a look at OR for our developing and emerging countries, we refer to http://ifors.org/ developing_countries/index.php/Main_Page.

Modern days' challenges in natural science and engineering, in economies and management, in societies and environments, in development and social complexity, in bio-, geo-, earth- and space-sciences, in OR and decision support systems are becoming more and more recognized and almost expected, everywhere in the world. Smart multidisciplinary discourse and joint creativity are urgently needed in order to find precise while at every moment stable solutions, to experience deep insights and contributions which are future-oriented, sustainable and impactful. They often initiate from real-world motivations, needs and inspirations, and along with premium and creative concepts and notions, metaphors and models, and bring us to powerful systems of control, optimization and decision support which are ready to provide effective and efficient agendas of managerial, organizational, economical, financial, environmental, political, creational and re-creational decisions-locally and globally. This process of creativity, analysis, innovation, invention and construction requires both intelligent networks, disciplinary and interdisciplinary communication, their neuronal, cognitive, communicational, social and practical dynamics and stimulation. Interdisciplinary collaborations and networks are based on curiosity and freedom, respect and commitment. In all of these items, modern Optimization and Optimal Control, Data Mining, Machine Learning, OR and AI come into action and service as key technologies of model selection, modeling, regularization and of careful pre- and post-processing, of design and preparation, of guidance and interest, of continuous concern, trust and responsibility.

We invited papers which in scientific, applied and creative ways are inspiring and helpful in our modern life and upcoming crises or provide a rigorous mathematical analysis of models where tools from OR, Mathematical Modeling, Statistical Learning, Optimization, Data Science, Heuristics, AI and other research areas of social and economic, biological and life-scientific, physical, energetic and informational sciences. These papers should have a positive impact on sustainable development in our rural countryside and our cities, in our oceans and the sky, for mobility and communication, understanding and empathy, collaboration and peace, inside and among our countries and nations, our human beings and creatures in the world.

\footnotetext{
1 Poznan University of Technology, Poznan, Poland

2 Institute of Applied Mathematics, Middle East Technical University, Ankara, Turkey

3 UOW Malaysia, KDU Penang University College, Petaling Jaya, Malaysia

4 Facultad de Ingeniería, Universidad Panamericana, Mexico City, Mexico

5 Parthenope University and at Federico II University, Naples, Italy

6 Nuevo Leon State University, San Nicolás de los Garza, Mexico

7 MERLIN Research Centre, Ton Duc Thang University, Ho Chi Minh City, Vietnam
} 
In the first paper, Shuang Cai and Ke Liu investigated "Online Scheduling on Two Parallel Identical Machines Under a Grade of Service Provision”, considering three different versions: the two machines cannot be idle at the same time until all arrived jobs have been processed; further to the first problem, jobs are processed on a firstcome, first-serviced basis; every job must be assigned to one of the two machines as soon as it arrives.

In the second paper, Timothy Ganesan, Pandian Vasant and Igor Litvinchev demonstrated "Chaotic Simulator for Bilevel Optimization of Virtual Machine Placements in Cloud Computing". Comparative analysis on the performance was carried out. A new "cascaded hypervolume" indicator is introduced and applied to measure the dominance of the solutions produced by both methods. Detailed analysis on the computational results is offered.

In the third paper, Subrata Panja and Shyamal Kumar Mondal presented "Analytics of an Imperfect Four-Layer Production Inventory Model Under Two-Level Credit Period Using Branch-and-Bound Technique". They aim to find the optimal number of production and business cycles of the manufacturer such that the integrated system can get the maximum profit. The model is elaborated in a numerical example, and a sensitivity analysis is done.

In the fourth paper, Diriba Kajela Geleta and Mukhdeep Singh Manshahia studied "A Hybrid of Grey Wolf Optimization (GWO) and Genetic Algorithm (GA) for Optimization of Hybrid Wind and Solar Renewable Energy System". The result of their algorithm is compared with the results of iteration method, GWO, GA, artificial bee colony and particle swarm optimization techniques. The authors' results are better when compared with those mentioned in the text.

In the fifth paper, Zahra Behdani and Majid Darehmiraki introduced "An Alternative Approach to Rank Efficient DMUs (decision-making units) in DEA (data envelopment analysis) via Cross-Efficiency Evaluation, Gini Coefficient and Bonferroni Mean". The proposed method breaks the tie between efficient decision-making units. A numerical example and real application of this method are offered in the ranking of research and development investment companies of pharmaceutical and biotechnology industries.

In the sixth paper, Lin-Lin Wang, Li-Wei Liu, Xu-Dong Chai and Zhen Wang studied "On Equilibrium in a Constant Retrial Queuing System with Reserved Time and Vacations", in the fully unobservable case. Steady-state condition of the system is analyzed by Foster's criterion, and the customers' expected waiting time is obtained. Equilibrium strategies of customers and socially optimal strategy are derived, and effects of some main system parameters are studied.

In the seventh paper, Tham Vo and Phuc Do presented "TKES (Trendy Keyword Extraction System): A Novel System for Extracting Trendy Keywords from Online News Sites", used for detecting bursts. Results from user requests could be displayed visually. They create a method to find a trendy keyword set which is defined as a set of keywords that belong to the same burst. This work also describes the datasets used for our experiments, processing speed tests of the two new algorithms.

In the eighth paper, Evgeniia Markova, Inna Sidler and Victor Trufanov analyzed "Optimization Problem for the Integral Model of Developing Systems". An algorithm for solving of the optimal control problem is developed. The influence of economic indices on the solution to the optimal control problem is studied. Using real-life data, 
calculations are carried out for the optimal development of the Unified Energy System of Russia until 2050.

In the ninth paper, B. Bazeer Ahamed and Murugan Krishnamoorthy offered "SMS (short message service) Encryption and Decryption Using Modified Vigenere Cipher Algorithm". Their algorithm's advantage is that finding factors for the large composite numbers is difficult. Rivest-Shamir-Adleman algorithm is combined with Vigenere cipher so that the new algorithm is secured and consumes less time than Rivest-ShamirAdleman. This approach is widely employed to encrypt SMS.

In the tenth paper, Long The Nguyen, Huong Thu Nguyen, Alexander Diomidovich Afanasiev and Tao Van Nguyen introduced "Automatic Identification Fingerprint Based on Machine Learning Method". Identification system consists of the following steps: improving image quality and image segmentation to identify the fingerprint area, extracting features and matching the database. Accuracy reached $97.75 \%$ on a mixed quality fingerprint database.

In the eleventh paper, Mitra Movassaghi and Soroush Avakh Darestani presented "Multiple Cross-Docks Scheduling with Multiple Doors using Fuzzy Approach and Metaheuristic Algorithms". A fuzzy multi-objective LP model is offered to solve the transportation decision-making with fuzzy objective function coefficients. This model is NP-hard. The authors applied metaheuristic algorithms like genetic and ant colony in order to solve our proposed problem.

This JORSC special issue offers enlightening contributions for research scholars and the emerging youth from across our planet in the research areas of OR and its novel modeling, optimization, statistical, computer science and AI approaches, along with the cutting-edge results, methods and applications. This JORSC special issue could not have been organized without the strong support and help from the Editors, Experts and Coworkers of JORSC and Springer. We as the Guest Editors hope that the selected topics display a core choice of international scholarly research on the emerging and complex problems of modeling, simulation, and optimization in operational research. We are very thankful to JORSC and Springer publishing house for the honor of accommodating this special issue as a pioneering science project. Special thanks are extended to the Editor in Chief and further Editors of JORSC, for their interest and confidence, facilitation and help donated to us and our special issue since the first moment of our endeavor, and to the editorial office for its steady advice and guidance, in every regard. We thank all the authors because of their hard and intelligent work, and for their readiness to share their newest achievements with our entire community. Now, we very much hope that their findings and offers will inspire for cooperation and advancements on a worldwide and highest stage.

The guest editors would like to sincerely thank the authors of ICO 2018 (https:// www.icico.info) for their marvelous contribution in submitting their novel research works.

August 1st, 2021 